\title{
Aprimoramento cognitivo e uso de substâncias: um estudo em torno da divulgação midiática brasileira sobre "smart drugs" e nootrópicos
}

\author{
Bruno Castro1 \\ Elaine Reis Brandão²
}

Resumo

O consumo de fármacos com o propósito de otimizar processos mentais, como memória, concentração e estado de alerta, tem se expandido, principalmente mediante disseminação pela internet. As chamadas "smart drugs" e fármacos nootrópicos são utilizados com a expectativa de obtenção de melhor desempenho em tarefas profissionais e acadêmicas. O objetivo deste artigo foi analisar a difusão do uso de medicamentos para aprimoramento cognitivo, a partir de publicações midiáticas brasileiras. A metodologia adotada foi a pesquisa qualitativa documental, baseada em materiais de divulgação científica que compreendem reportagens publicadas entre os anos de 2009 e 2017, em veículos de mídia reconhecidos nacionalmente. Na propagação de saberes e práticas relacionadas à otimização do desempenho cognitivo pelo uso de substâncias, a internet constitui uma tecnologia midiática potencial para os veículos de divulgação, na disseminação e socialização de tais substâncias. A construção de saberes, noções e representações sociais relacionadas ao tema abordado desafia as instâncias de controle hegemônico do cuidado em saúde. Na esteira dos processos de farmacologização da sociedade, o tratamento público do tema permite estabelecer uma analogia com a "questão das drogas" tornadas ilícitas na contemporaneidade.

Palavras-chave: medicalização; nootrópicos; aprimoramento biomédico.

\section{Cognitive enhancement and substance use: a study around Brazilian media dissemination on smart drugs and nootropics}

Abstract

The use of drugs to improve mental processes, such as memory, concentration and alertness, has been expanding through the Internet. The so-called smart drugs and nootropic drugs are used with the expectation that better performance can be achieved in professional and academic tasks. The aim of this article was to analyze the diffusion of cognitive enhancement drugs' use from Brazilian media publications. The methodology adopted was the qualitative documentary research, based on scientific dissemination materials that include reports published between 2009 and 2017, in nationally recognized media outlets. In the propagation of knowledge and practices related to the optimization

1 Doutorando em Saúde Coletiva pelo Instituto de Estudos em Saúde Coletiva (IESC) da Universidade Federal do Rio de Janeiro (UFRJ)

2 Professora Associada do Instituto de Estudos em Saúde Coletiva (IESC) da Universidade Federal do Rio de Janeiro (UFRJ) 
of cognitive performance through substance use, the Internet constitutes a potential media technology as a mean for the dissemination and socialization of such substances. The construction of knowledge, notions and social representations related to the theme addressed challenges the hegemonic control instances of health care. In the wake of the pharmacologization processes of society, the public treatment of the subject makes it possible to draw an analogy with the "drug issue" made illicit in contemporary times.

Keywords: medicalization; nootropic agents; biomedical enhancement.

\section{Introdução}

O desenvolvimento do conhecimento científico, paralelo à expansão da indústria farmacêutica, aliado à consolidação do que muitos autores chamam de complexo médicoindustrial (CLARKE et al, 2010; MIGUELOTE; CAMARGO JR, 2010), têm uma importância crucial na remodelação das relações sociais por meio das quais tentamos compreender a nós mesmos, nas sociedades ocidentais contemporâneas. A história da medicina tem sido ligada à história das formas com que os seres humanos têm tentado se tornar melhores do que são (ROSE, 2013). As sociedades contemporâneas introduziram um horizonte de aprimoramento constante e uma necessidade permanente de melhoria de nossas capacidades (BARROS; ORTEGA, 2011; ORTEGA et al, 2010).

Uma questão que atualmente chama atenção é a promessa de que fármacos e suplementos alimentares possam ser utilizados para aumentar o estado de alerta, concentração, memória e outros aspectos do funcionamento cognitivo, configurados num recurso que podemos denominar "aprimoramento cognitivo". O consumo de substâncias para aprimorar a performance dos processos mentais/ neurocognitivos em indivíduos saudáveis tem objetivos específicos que, em grande medida, residem na expectativa de se obter maior eficiência e melhor desempenho em tarefas profissionais e acadêmicas. As chamadas "smart drugs" ou fármacos nootrópicos têm se expandido crescentemente mediante difusão pela internet, diante da divulgação de substâncias voltadas para a otimização do desempenho cognitivo. Uma série de relatórios e artigos já surgiram em resposta a estes desenvolvimentos, abordando as implicações éticas, jurídicas e sociais dos medicamentos para aprimoramento cognitivo e o grau em que devem ser regulamentados (WILLIAMS et al, 2011). Com o objetivo de melhorar o desempenho acadêmico e profissional, não uma nova era de fármacos, mas medicamentos antes comercializados têm sido utilizados fora das indicações para os quais foram aprovados, em usos off-label ${ }^{3}$, em grande medida por indivíduos que não buscam o tratamento de quaisquer sintomas. Muitos fármacos utilizados no tratamento de condições específicas de saúde mental, tais como o metilfenidato (Transtorno do Déficit de Atenção e Hiperatividade TDAH), donepezil (Alzheimer) e modafinil (narcolepsia) estão sendo utilizados por pessoas saudáveis para aperfeiçoar o desempenho cognitivo (WILLIAMS et al, 2011). Ademais, empresários e companhias diversas também buscam a produção do seu "viagra para o cérebro" e alguns já os prometem pela comercialização na internet (ROSE, 2013). As vendas internacionais de suplementos para cognição, excluídos os medicamentos anteriormente citados, excederam 1 bilhão de dólares por ano em 2015 e se mantêm em crescimento (CHINTHAPALLI, 2015). Nos Estados Unidos, estima-se que as vendas desses suplementos tenham atingido 217 milhões de dólares em 2018, com um aumento de mais de $40 \%$ se comparadas ao ano de 2017 (CHAKER, 2019).

Na página eletrônica do Portal Regional da Biblioteca Virtual em Saúde (BVS), no atalho destinado à consulta dos Descritores em Ciências

3 Constituem o uso de medicamentos para uma indicação não aprovada, um grupo etário não aprovado, uma dose não aprovada ou uma forma de administração não aprovada, de acordo com o registro sob o qual um fármaco é licenciado no órgão sanitário competente (FREITAS; AMARANTE, 2015). 
da Saúde (DECS), é possível encontrar a expressão "aprimoramento biomédico" como um descritor de assunto para a localização de publicações relacionadas. A página apresenta dois sinônimos para a expressão: "aperfeiçoamento biomédico" e "reforço biomédico", e a define como "Uso de intervenções baseadas na tecnologia para melhorar a capacidade funcional, em vez de tratar a doença" (BVS, 2019a). Na mesma página, é possível encontrar o termo "nootrópicos" com a seguinte definição: "Fármacos usados para facilitar especificamente o aprendizado ou a memória, particularmente para impedir os déficits cognitivos associados às demências". (BVS, 2019b).

Esse termo corresponde, na BVS, à expressão "cognitive enhancers", frequentemente utilizada em artigos publicados na língua inglesa para se referir às substâncias utilizadas com a finalidade de aprimoramento cognitivo cognitive enhancement ou neuroenhancement - principalmente nos Estados Unidos, Canadá e Europa, onde a discussão sobre esses usos é recorrente (BATTLEDAY; BREM, 2015; FARAH, 2015; FORLINI; GAUTHIER; RACINE, 2013; SAHAKIAN; MOREIN-ZAMIR, 2015). Tais substâncias também são representadas pela expressão "smart drugs" (CAKIC, 2009), entre as quais são agrupados aqueles fármacos utilizados em saúde mental que, pela legislação brasileira, constituem medicamentos sujeitos a controle especial e necessitam de receitas específicas, que são retidas no ato de dispensação ou fornecimento ${ }^{4}$.

O objetivo deste artigo é propor uma discussão sobre esses fármacos e suas formas de divulgação e socialização. O próprio contexto sociocultural em que essas práticas se propagam diz algo sobre a forma como o fenômeno se apresenta sob uma ética competitiva e individualista característica das sociedades ocidentais contemporâneas. Além disso, toda a compreensão sobre a corporalidade humana baseada no fisicalismo aponta para o entendimento do sistema nervoso como circuitos neuroquímicos absorvidos no desenvolvimento de uma psicofarmacologia apta a possibilitar o aparecimento de tecnologias bioquímicas voltadas à otimização das funções cerebrais (DUARTE, 1999; AZIZE, 2008). Os limites da fronteira entre tratamento e aprimoramento se mostram cada vez mais nebulosos dentro da variabilidade do que pode ser considerado um ou outro ao longo do tempo e é cada vez mais difícil responder o que é "normal", "deficiente", o que é "ser melhor" e o que deve ser submetido a intervenções terapêuticas (GAUDENZI, 2017; ROSE, 2013). A propagação do aprimoramento cognitivo com o uso de substâncias e sua popularização pela internet desafiam as instâncias de controle hegemônico de cuidado em saúde, apesar da ampla disseminação do tema no senso comum. Se considerarmos a importância da Internet como campo aberto para a difusão de informações e construção de conhecimento, então estamos diante de um potencial incomparável para reconfiguração desses saberes que não seria possível além da comunicação mediada pelas mídias digitais.

Ao observar as discussões engendradas perante os paradigmas éticos, legais e sociais, assim como as oposições que são suscitadas a respeito de tais substâncias e seus usos para aprimoramento cognitivo (ORTEGA et al, 2010), é possível estabelecer certa analogia com a "questão das drogas" tornadas ilícitas, principalmente no que diz respeito ao tratamento público do tema, baseado em grande medida nos eixos da medicalização, da criminalização e da moralização (FIORE, 2006). Neste sentido, a noção de dispositivo trazida por Foucault (2006) lança luz para a compreensão do conhecimento produzido acerca dessas práticas, seus respectivos atores e as relações de poder engendradas (VARGAS, 2008). As relações ambivalentes que as sociedades contemporâneas mantêm com as drogas - no sentido mais amplo do termo -, marcadas pela repressão e pela incitação ao consumo, se coadunam com a expansão contemporânea do desenvolvimento de fármacos e todo o conhecimento advindo

4 De acordo com a Portaria n.344 de 1998 da Secretaria de Vigilância Sanitária (SVS), do Ministério da Saúde. 
da chamada "invasão farmacêutica" (VARGAS, 2008), uma vez que tal expansão propiciou a partilha moral entre drogas de uso lícito e drogas de uso ilícito, mais especificamente entre drogas, medicamentos, condimentos, alimentos e cosméticos. É nessa partilha que encaramos de um lado a criminalização das drogas tornadas ilícitas e de outro, o processo de invasão farmacêutica. É a partir desse ponto de vista que devemos analisar as relações existentes entre a proibição de determinadas substâncias psicoativas e a promoção de outras e em que medida esses sistemas se realimentam (COHEN et al, 2001). Nas relações de poder suscitadas a partir da geração e circulação de diferentes discursos acerca dos medicamentos e seus efeitos, o consumo não terapêutico do que antes eram medicamentos aprovados para o tratamento de determinadas condições se confunde com o uso medicamentoso ou não de substâncias tornadas ilícitas. Assim, o dispositivo das drogas cristaliza a tensão existente entre drogas de uso lícito e drogas de uso ilícito. As mesmas substâncias se polarizam de um ponto ao outro e o consenso se confunde na nebulosidade das fronteiras postas em questão.

\section{Metodologia}

Os métodos qualitativos de pesquisa não buscam a mensuração de fenômenos em grandes grupos, mas sim entender o contexto onde algum fenômeno ocorre, o que permite a observação de vários elementos simultaneamente em um pequeno grupo e um conhecimento aprofundado de um evento (VÍCTORA et al, 2000). Em sua aplicação à área da saúde, a metodologia qualitativa busca compreender o significado individual ou coletivo de um evento na vida das pessoas. Uma vez que, em torno do que as coisas significam, as pessoas organizarão de certo modo suas vidas, incluindo seus próprios cuidados com saúde (TURATO, 2005). Nesse sentido, as representações sociais, como formas de conhecimento socialmente elaboradas e partilhadas por um grupo, funcionam como orientadoras das práticas sociais. Também podem ser encaradas como uma lógica que une as diferentes percepções individuais de um grupo (VÍCTORA et al, 2000). Como campo de pesquisa, a internet é um espaço diferenciado para a compreensão dos comportamentos de indivíduos e grupos, em que os conteúdos gerados e propagados por consumidores e produtores dessas informações abrem a possibilidade para criação de novas formas de sociabilidade e interação social. Ademais, constitui um campo aberto para a captação de documentos passíveis de serem fontes para a pesquisa e o tratamento analítico. A disponibilidade das informações, a postura ativa dos indivíduos e a facilidade de acesso constituem oportunidades para dispersão e produção de conhecimento no ambiente virtual, que se configura como tecnologia midiática potencial.

O presente estudo utilizou a metodologia qualitativa, em sua abordagem documental (SÁ-SILVA et al, 2009), sob uma perspectiva socioantropológica, para captar as representações e práticas sobre o uso de medicamentos para aprimoramento cognitivo, a partir de reportagens publicadas em sites de veículos de mídia reconhecidos nacionalmente. Assim, o acesso ao objeto de estudo ocorreu por intermédio da observação e análise de documentos em páginas da internet com veiculação pública de medicamentos para aprimoramento cognitivo no país. O material empírico foi coletado em fontes de domínio público na internet, o que incluiu páginas/sítios com endereço eletrônico, reportagens e vídeos publicados e relacionados ao tema. Como ferramenta para a coleta de dados, a pesquisa documental utiliza métodos e técnicas para a apreensão e compreensão de documentos dos mais variados tipos. Recorre principalmente a fontes primárias, ou seja, materiais que ainda não receberam tratamento analítico (SÁ-SILVA et al, 2009). A análise documental favorece a observação de indivíduos, grupos, conceitos, conhecimentos, comportamentos, mentalidades, práticas, entre outros (CELLARD, 2008). Embora não seja possível restringir as representações acerca do debate público sobre o assunto aos meios de comunicação de massa, é importante 
considerar a difusão e a produção de discursos científicos sobre as drogas, num sentido mais amplo, assim como a importância dos saberes biomédicos para a abordagem do tema que, apesar da complexidade dos saberes e lógicas que o engendram, alcançam certa difusão no chamado senso comum (FIORE, 2006).

$\mathrm{Na}$ etapa exploratória deste estudo, foi realizada uma busca na página eletrônica do Google com a expressão "melhorar desempenho cognitivo". Nos primeiros resultados, entre páginas que forneciam informações sobre atividades, atitudes e exercícios para maximizar o desempenho cognitivo, uma página em português da agência britânica de notícias BBC trazia uma reportagem sobre substâncias que supostamente funcionariam como "potencializadores cognitivos" e que estariam sendo utilizadas por trabalhadores do Vale do Silício, na Califórnia. Tais substâncias recebiam o termo "nootrópicos" para classificá-las de acordo com a capacidade de otimizar o desempenho mental. Voltando à página do Google, a inserção do termo "nootrópicos" no mecanismo de buscas trouxe como resultado uma infinidade de páginas contendo informações sobre tais substâncias, dentre as quais são descritos suplementos alimentares e medicamentos normalmente prescritos para tratamento de determinadas condições de saúde mental. A partir de então, decidi fazer um recorte dentre a infinidade de substâncias utilizadas para tal fim, para inicialmente compreendê-las melhor e, em seguida, reunir e categorizar as informações obtidas sobre tais medicamentos.

Para verificar a ocorrência do aprimoramento cognitivo farmacológico em mídias de grande circulação nacional, o material empírico foi coletado entre os meses de agosto e outubro de 2017, com os termos "smart drugs", "nootrópicos", "drogas da inteligência" e "pílulas da inteligência" nos acervos digitais das revistas Superinteressante e Veja e dos jornais Folha de São Paulo e Extra. Também foram pesquisados os portais da internet G1, globo.com e R7. Além disso, os termos acima foram pesquisados ainda a partir do mecanismo de buscas do Google, à procura de notícias publicadas em veículos reconhecidos nacionalmente, relacionadas ao tema do aprimoramento cognitivo farmacológico. Selecionou-se reportagens que citavam a utilização de substâncias com o objetivo de otimização do desempenho cognitivo. Foram coletadas 23 publicações nos canais de mídia selecionados, referentes ao período entre 2009 e 2017. Dessas, dois textos foram repetidos em veículos diferentes, o que resultou no total de 21 publicações, 19 de jornais e revistas e duas veiculadas na televisão.

\section{Resultados e discussão}

Nas reportagens selecionadas para a pesquisa, além dos termos utilizados nas buscas, apareceram as denominações "aprimoradores cerebrais", "aprimoradores cognitivos", "viagra do cérebro", "brain drugs", "rebite universitário" e "droga inteligente", todas como referência às substâncias utilizadas para aprimoramento cognitivo. Pesquisas anteriores identificaram denominações como "drogas para turbinar o cérebro", "droga de inteligência" e "droga da obediência”, utilizadas pela mídia para descrever esses usos (BARROS; ORTEGA, 2011; COELHO; LEAL, 2015). Em território nacional, o medicamento mais conhecido para esses fins é o metilfenidato, que ficou popularizado por sua vinculação ao diagnóstico de TDAH e devido à ampliação dos critérios para inclusão diagnóstica do TDAH (ORTEGA et al, 2010). A indissociabilidade TDAH-metilfenidato gerou um aumento no consumo do medicamento, na esteira dos espaços de expansão médica e psiquiátrica ao longo de algumas décadas (ORTEGA et al, 2010; ROSE, 2013).

Ortega et al (2010) analisaram as publicações brasileiras, científicas e de mídia popular, sobre a Ritalina - marca sob a qual é comercializado o metilfenidato. Em sua investigação, confirmaram que os artigos científicos abordam o TDAH e consideram o medicamento imprescindível no tratamento do transtorno. O tema do uso terapêutico do medicamento para tal categoria diagnóstica predomina em ambos os tipos de 
publicação. A produção científica nacional sobre os usos do metilfenidato está em grande medida vinculada às pesquisas sobre TDAH no Brasil (ORTEGA et al, 2010; ESCHER; COUTINHO, 2017). Essa pesquisa (ORTEGA et al, 2010), realizada em 2009, verificou que, de 72 reportagens publicadas em jornais e revistas de maior tiragem nacional, entre 1998 e 2008, apenas duas tratavam dos usos "não médicos" do fármaco para melhorar o desempenho cognitivo.

Dos documentos selecionados para a presente pesquisa, apenas uma reportagem foi encontrada para o ano de 2009 e duas no ano de 2011. Para os anos de 2012, 2013 e 2014, foram encontradas duas publicações por ano. $\mathrm{O}$ ano com mais publicações foi 2015, com cinco reportagens. $\mathrm{O}$ ano de 2016 teve três publicações e o de 2017, quatro publicações. O conjunto dos textos jornalísticos encontra-se listado no Anexo, em fontes consultadas. É possível verificar um aumento nas publicações de mídia popular ao longo dos anos, se compararmos os dados atuais com aqueles da pesquisa que analisou os anos de 1998 a 2008 (ORTEGA et al, 2010). Na presente pesquisa, a utilização em ambientes de estudo foi a justificativa mais comum, sendo verificada em 13 dos 21 documentos. São estudantes em preparação para vestibular e concursos públicos e universitários. Esse dado reitera a abrangência das pesquisas científicas já realizadas em outros países, que documentam o uso destas substâncias em indivíduos saudáveis e abrange estudantes secundaristas e universitários, havendo alguns estudos que estimaram as prevalências de tais usos nessas populações (BATTLEDAY; BREM, 2015; FARAH, 2015; SAHAKIAN; MOREINZAMIR, 2015).

Em menor grau, em oito das 21 publicações, são apontados ambientes de trabalho competitivos e com nível elevado de exigência, como no caso de militares, médicos e trabalhadores do mercado financeiro. Os fármacos mais reportados em ambas as situações são aqueles sujeitos a controle especial, metilfenidato e modafinil, citados em 12 e dez reportagens, respectivamente. Outros da mesma categoria que aparecem são lisdexanfetamina ${ }^{5}$, quatro vezes, e donepezil ${ }^{6}$, duas. Também são mencionados o piracetam (medicamento que não possui controle especial pela legislação brasileira) e seus derivados (substâncias que não possuem registro na ANVISA - Agência Nacional de Vigilância Sanitária) em seis textos.

Das 21 reportagens coletadas, sete tratavam de substâncias de classificações diferentes perante à ANVISA - medicamentos sujeitos a controle especial, medicamentos vendidos sem retenção da receita e suplementos alimentares - numa mesma categoria, a partir da finalidade de uso. Apenas duas faziam uma diferenciação não por essas classificações, mas pela ausência de efeitos colaterais significativos, pelo baixo potencial para causar dependência e pela baixa toxicidade. Essas são as características principais utilizadas na década de 1970, pelo médico e pesquisador da indústria farmacêutica Corneliu Giurgea (1982), para enumerar o perfil da classe dos nootrópicos. Tal caracterização reforça o perfil de baixa incidência de graves efeitos adversos em comparação com os fármacos psicotrópicos lançados no mercado até então (GIURGEA, 1982). O fato de os nootrópicos não possuírem efeitos farmacológicos expressivos e, potencialmente, não causarem dependência, os diferencia dos medicamentos de uso controlado que, dessa forma, não se encaixam completamente nos critérios de definição dos nootrópicos (GIURGEA, 1982). Esses seriam considerados como outro grupo dentro do universo mais amplo das smart drugs, apesar de, na atualidade, a classe dos nootrópicos ser mais relacionada à finalidade de uso para aprimoramento cognitivo do que propriamente com uma classe de medicamentos com características bioquímicas semelhantes.

Em 15 das 21 publicações, a opinião de especialistas da área biomédica marcava o perfil de alerta das reportagens quanto à segurança e

5 Comercializado no Brasil sob o nome Venvanse ${ }^{\varpi}$, para o tratamento de TDAH (Transtorno de Déficit de Atenção e Hiperatividade). Fármaco sujeito a controle especial pela ANVISA.

6 Fármaco utilizado no tratamento da Doença de Alzheimer e sujeito a controle especial pela ANVISA. 
o risco da utilização de fármacos com objetivo de otimização do desempenho cognitivo. Essa tendência confirma a legitimidade conferida ao argumento de autoridade dos saberes biomédicos no tratamento da "questão das drogas" na contemporaneidade, sejam estas lícitas ou ilícitas (FIORE, 2006).

Dois influentes pesquisadores do aprimoramento cognitivo, os neurocientistas Ruairidh Battleday e Anna-Katharine Brem, das universidades de Oxford e Harvard, respectivamente, afirmaram recentemente, numa entrevista ao Instituto Humanitas Unisinos ${ }^{7}$, que smart drugs são substâncias com a função de aumentar ou ampliar as funções cognitivas em pessoas saudáveis, sem causar efeitos colaterais significativos. Os pesquisadores acenam para a possibilidade de o modafinil - um medicamento sujeito a controle especial - corresponder a essa classificação, apesar das ressalvas sobre a necessidade de mais informações sobre seus efeitos colaterais em indivíduos saudáveis e sobre seus efeitos a longo prazo. Tais afirmações tornam ainda mais problemático algum consenso sobre as denominações e as categorizações dessas substâncias quanto às finalidades de uso para aprimoramento cognitivo.

Parcela considerável das reportagens apresentou considerações sobre a segurança e a eficácia das substâncias abordadas. Em 16 textos, as observações sobre possíveis efeitos colaterais e a longo prazo são consideradas. Em 15 destes, a opinião de especialistas corrobora tais ponderações, como na reportagem exibida no programa televisivo Fantástico ${ }^{8}$, da Rede Globo, em 05 de junho de 2016, ao afirmar que o tema do aprimoramento cognitivo pelo uso de medicamentos e suplementos alimentares tem se tornado recorrente, principalmente em contextos individualistas e competitivos, como os de grande concorrência em concursos públicos no Brasil. Nela, o psiquiatra Dartiu Xavier, da UNIFESP, afirma que todo medicamento oferece algum risco de ocorrência de efeitos colaterais e que os nootrópicos, medicamentos com a finalidade de proteger o cérebro em indivíduos que sofreram danos neurológicos decorrentes de doenças como Alzheimer ou derrames cerebrais, só seriam eficazes quando há deficiências como essas. Em outra reportagem, exibida no programa Saia Justa ${ }^{9}$, do canal televiso fechado GNT, em 14/05/2013, o neurocientista Carl Hart, da Universidade de Columbia, é taxativo ao salientar que é preciso estudar os efeitos a longo prazo do medicamento modafinil, pois ainda existem poucas informações a esse respeito. Os possíveis benefícios clínicos da utilização de medicamentos para essa finalidade em indivíduos saudáveis não são bem comprovados pela literatura científica contemporânea (FARAH, 2015; FORLINI; GAUTHIER; RACINE, 2013; SAHAKIAN; MOREIN-ZAMIR, 2015; BATISTELA, 2011).

Ao caracterizar o uso não medicamentoso e, em grande medida, off-label dessas intervenções farmacológicas, é preciso considerar as formas contemporâneas de utilização dos medicamentos e as interações desses objetos nas redes de significados individuais $\mathrm{e}$ coletivos nas quais estão inseridos (COHEN et al, 2001). É nesse sentido que se expressa a importância que os medicamentos exercem para a construção de identidades coletivas a partir do compartilhamento de experiências relacionadas ao consumo de tais substâncias. A produção de modos de subjetividade, a partir dos discursos de verdade baseados no saber biomédico, permite a adoção de identidades a partir da manipulação de estados corporais pela via farmacológica (CLARKE et al, 2010; COLLIN, 2016; ROHDEN, 2017; CASTRO, 2020). Nessa direção, é importante ressaltar que muitas páginas da internet podem ser

7 
simplesmente veículos de empresas comerciais, interessadas em divulgação de medicamentos, de novas tecnologias, ou mesmo valores morais que levem os usuários a buscarem seus produtos (PEREIRA NETO et al, 2015). O nível crescente de atenção pública para o assunto, apesar de um corpo limitado de evidências científicas é uma questão considerável nesse contexto. Pesquisas anteriores demonstraram que a mídia pode desempenhar um papel na disseminação de informações, estimulando $\mathrm{o}$ interesse $\mathrm{e}$ reforçando a deturpação do nível de evidência (WADE; FORLINI; RACINE, 2014). Ademais, a disseminação cultural dos consumos de performance, principalmente entre a população jovem, constitui um fenômeno cuja amplitude vai além das práticas de consumo, haja vista o efeito de visibilidade e familiaridade pública proporcionado pelos veículos midiáticos a estes meios terapêuticos e seus usos de performance. No entanto, o acesso público a tais recursos não se transforma em adesão ao seu consumo somente pelos mecanismos sociais de difusão. Tal conversão advém da confluência de outros mecanismos sociais, revelando a importância dos próprios contextos cotidianos dos indivíduos (LOPES; RODRIGUES, 2015; COELHO; LEAL, 2015). De todo modo, as mesmas substâncias psicoativas podem, então, estar disponíveis em contextos variados de consumo, mesmo que se configure uma partilha moral que conforma discursos públicos específicos entre o estatuto social de drogas de uso lícito e drogas de uso ilícito (VARGAS, 2008; FIORE, 2006). Apesar das questões sobre risco e segurança de tais intervenções farmacológicas, suscitadas pelas reportagens consultadas, a crescente atenção pública dedicada ao tema demonstra a existência de um espaço propício para a transformação de condições, capacidades e potencialidades humanas em oportunidades para intervenções farmacológicas, na definição de um processo de farmacologização da vida cotidiana (WILLIAMS et al, 2011). Como dito pelos sociólogos Nick J. Fox e Katie J. Ward:

o sucesso de uma droga reside não somente na sua capacidade de atingir um efeito, mas também na sua interação com forças culturais e sociais que definem uma condição como justificada por resoluções farmacológicas (FOX; WARD, 2008, p.862, tradução nossa).

Trata-se de ponderar a eficácia simbólica (LÉVI-STRAUSS, 1996) que esse processo abarca a partir do que possa ser considerado efeito placebo e o que este representa na eficácia geral da droga, ao compor com o efeito químico da substância (PIGNARRE, 1999). Por outro lado, a construção da eficácia também passa pelo agenciamento dos sujeitos e pela forma como os saberes biomédicos são reconstruídos a partir das experiências pessoais e coletivas relacionadas à interação entre essas drogas e os corpos dos indivíduos que as utilizam (FLEISCHER, 2012). $\mathrm{Na}$ relação com os contextos em que estão inseridos, os medicamentos são constantemente interpretados e socializados, reconstruídos e reelaborados, para além das moléculas que os constituem (HARDON; SANABRIA, 2017). Para além desse eixo de análise, o conceito de dispositivo, resgatado pelo antropólogo Eduardo Vargas (2008), a partir da noção trazida por Foucault (2006), lança luz sobre as relações ambivalentes que as sociedades contemporâneas mantêm com as drogas - no sentido mais amplo do termo -, marcadas pela repressão e pela incitação ao consumo e cristalizadas nesta espécie de dispositivo das drogas (VARGAS, 2008). O conjunto de noções, saberes, discursos e representações sociais que conforma o discurso público sobre drogas - no sentido mais amplo do termo - se fundamenta em grande medida pelo eixo medicalizante (FIORE, 2006). Apesar das constantes ressalvas das autoridades médicas acerca do risco e segurança desses usos, caracterizados como intervenções farmacológicas, os conhecimentos sobre o aprimoramento cognitivo com o uso de fármacos ultrapassam os domínios dos atores oficiais do cuidado em saúde, sejam médicos ou outros profissionais de saúde, haja vista a crescente divulgação midiática sobre o tema. Ademais, os saberes e práticas intersubjetivas 
vêm consolidar uma base de conhecimentos que direcionariam os usuários para a obtenção dos efeitos esperados. Nessa direção, a noção de dispositivo é útil para abordar como substâncias catalisam novas formas de sociabilidade $\mathrm{e}$ subjetividade, seja a partir de usos não médicos ou ilícitos, como no caso dos fármacos para aprimoramento cognitivo, seja no tratamento de condições com fármacos psicotrópicos. Um bom exemplo pode ser visto com as metanfetaminas, a partir do exemplo do Pervitin. O que na década de 1950 era considerado um fármaco socialmente aprovado para aprimoramento cognitivo, tornase uma substância ilícita no Brasil, com seu princípio ativo presente na lista de substâncias proscritas no país (ARAÚJO, 2015). A construção do conhecimento sobre o uso dessas substâncias - mesmo entre o público leigo - e as relações de poder entre os atores que articulam tais conhecimentos, a partir da compreensão, apreensão e propagação dos discursos relacionados, redefinem constantemente esses mesmos usos (CASTRO; BRANDÃO, 2020). Apesar dea maior partedassubstânciasatualmente utilizadas com fins de aprimoramento cognitivo não serem classificadas como ilícitas, "implicam constantemente as fronteiras da tolerância social e da licitude" (LABATE et al, 2016, p.21). Mesmo que a divulgação midiática desses usos tenha influência na construção desses conhecimentos, é importante compreender melhor como tais saberes contribuem para a abertura dos espaços sociais em que circulam (COLLIN, 2016). Nessa direção, o arcabouço da literatura antropológica que trata do tema das drogas no Brasil (ALVES; PEREIRA, 2019) pode fornecer importante contribuição para o aprofundamento do estudo sobre o uso de fármacos com finalidade de aprimoramento cognitivo, no cenário brasileiro, e considerar a necessária contextualização a partir dos marcadores específicos de diferenciação social que o caracterizam.

\section{Conclusão}

Nas práticas de aprimoramento cognitivo farmacológico, diversas substâncias têm sido designadas por aqueles que pretendem melhorar o desempenho de características do funcionamento cognitivo como concentração, atenção, memória e estado de alerta. No Brasil, as substâncias mais difundidas para esse fim são os derivados anfetamínicos. O metilfenidato, como tal, é o protagonista de diversos estudos que têm levantadoadiscussãosobreouso demedicamentos com essa finalidade. Outros fármacos da mesma classe também são historicamente associados à utilização como estimulantes, haja vista o exemplo das anfetaminas, mais conhecidas por serem prescritas como anorexígenos.

No Brasil, tais compostos são submetidos a um controle específico para o acesso pela população, motivo pelo qual são conhecidos como "medicamentos controlados" ou "tarja preta”. Esse grupo de medicamentos também é associado ao potencial para causar dependência física e/ou psíquica, além dos riscos relacionados ao seu uso contínuo, que envolvem condições cardiovasculares e transtornos mentais, no caso das anfetaminas. Além dessas, diversos outros fármacos de acesso menos controlado vêm sendo divulgados para utilização com fins de aprimoramento cognitivo.

A internet, como tecnologia midiática potencial, constitui o principal meio para difusão de "smart drugs" e nootrópicos, sem o qual seria difícil a propagação das informações sobre tais substâncias. Diante de contextos competitivos e individualistas característicos das sociedades ocidentais contemporâneas, a divulgação dessas substâncias constitui um fenômeno subjacente ao processo de farmacologização da sociedade e aponta para a importância dos veículos de divulgação midiática na disseminação $e$ socialização da possibilidade de aprimoramento cognitivo a partir do uso de recursos farmacológicos.

Em meio à distinção entre "smart drugs" e nootrópicos, que envolve a ponderação dos eventuais riscos e efeitos associados, as semelhanças entre o que, de um lado, constitui usos ilícitos de substâncias legais e, de outro lado, usos de substâncias ilícitas, se estreitam. Na propagação de saberes e práticas enredados, 
as evidências observadas permitem apontar para analogias que devem ser abordadas em estudos futuros e que vão além da partilha moral que separa o lícito do ilícito. Nesse sentido, as racionalidades que direcionam a gestão desses usos ainda têm muito a explicitar sobre o entendimento dessas práticas. $\mathrm{O}$ progresso do debate que envolve o uso drogas numa abordagem mais ampla, incluídos os medicamentos, depende das perspectivas trazidas pelos usuários, sem deixar de considerar o poder de agenciamento das próprias substâncias e os sentidos que lhes são atribuídos nos processos de socialização.

\section{Referências bibliográficas}

ALVES, Ygor; PEREIRA, Pedro Paulo. "Antropologia e a questão das drogas: um século de debates políticos e teóricos”. In: BIB (São Paulo), n. 90, p. 1-23, 2019.

ARAÚJO, Marcelo. "Pervitin instead of coffee? Change in attitudes to cognitive enhancement in the 50's and 60's in Brazil". In: Practical Ethics News (Blog). Oxford: University of Oxford, 2015.

AZIZE, Rogério. "Uma neuroweltanschauung? Fisicalismo e subjetividade na divulgação de doenças e medicamentos do cérebro". In: Mana, v. 14, n. 1, p. 7-30, 2008.

BARROS, Daniele.; ORTEGA, Francisco. "Metilfenidato e aprimoramento cognitivo farmacológico: representações sociais de universitários". In: Saúde e Sociedade, v. 20, n. 2, p. 350-362, 2011.

BATISTELA, Silmara.Efeitos daadministração aguda de diferentes doses do metilfenidato sobre a cognição de jovens saudáveis. 2011. 88 f. Dissertação (Mestrado em Ciências) - Escola Paulista de Medicina, Universidade Federal de São Paulo, São Paulo, 2011.

BATTLEDAY, Ruairidh; BREM, Anna-Katharine. "Modafinil for cognitive neuroenhancement in healthy non-sleep- deprived subjects: a systematic review". In: European Neuropsychopharmacology, v. 25, p. 1865-1881, 2015.

BIBLIOTECA VIRTUAL EM SAÚDE (BVS). Portal Regional da BVS: informação e conhecimento para a saúde. [S.l: s.n.], 2019a. Disponível em: <http://pesquisa.bvsalud.org/ portal/decs-locator/?lang=pt\&tree

\section{. Portal Regional da BVS: informação} e conhecimento para a saúde. [S.l: s.n.], 2019b. Disponível em: <http://pesquisa.bvsalud. org / portal/decslocator/ ? lang=pt\&tree $\mathrm{id}=\&$ term $=$ nootr $\% \mathrm{C} 3 \%$ B3picos\&tree

CASTRO, Bruno. Aprimoramento cognitivo e a produção de modos de subjetividade: um estudo sobre o uso de substâncias "nootrópicas" a partir de um blog brasileiro. In: Saúde Soc. São Paulo, v. 29, n. 1, e190936, 2020.

CASTRO, Bruno Pereira; BRANDÃO, Elaine Reis. Circulación de información sobre medicamentos y otras sustancias para aumentar el rendimento cognitivo: un estudio de un blog brasileño (2015-2017). In: Salud Colectiva, v. 16, e2514, 2020.

CAKIC, Vince. "Smart drugs for cognitive enhancement: ethical and pragmatic considerations in the era of cosmetic neurology". In: Journal of Medical Ethics, v. 35, n. 10, p. 611615, 2009.

CELLARD, André. A análise documental. In: POUPART, J. et al. A pesquisa qualitativa: enfoques epistemológicos e metodológicos. Petrópolis: Vozes, 2008.

CLARKE, Adele. et al. Biomedicalization: technoscience, health, and illness in the U.S. Durham, NC: Duke University Press, 2010.

COELHO, Eleonora; LEAL, Ondina. "Fabricando um corpo sem limites: a busca pelo sucesso profissional e o consumo de 
metilfenidato". In: Corpo e saúde na mira da antropologia: ontologias, práticas, traduções. MCCALlUM, C. A.; ROHDEN, F. (Org.). Salvador: EDUFBA: ABA, 2015.

COHEN, David. et al. "Medications as social phenomena". In: Health, v. 5, n. 4, p. 441-469, 2001.

COLLIN, Johanne. "On social plasticity: the transformative power of pharmaceuticals on health, nature and identity". In: Sociology of Health \& Ilness, v. 38, n. 1, p. 73-89, 2016.

CHAKER, Anne Marie. "Nootropic or not? Brain-booster business raises concerns". In: The Wall Street Journal, 04 abr. 2019. Disponível em: <https://www.wsj.com/aarticles/ nootropic-or-not-brain-booster-business-raisesconcerns-11554912086>. Acesso em: 23 out. 2019.

CHINTHAPALLI, Krishna. "The billion dollar business of being smart”. In: BMJ, n. 351, p.1-2, 2015.

DUARTE, Luis Fernando. "O império dos sentidos: sensibilidade, sensualidade e sexualidade na cultura ocidental moderna”. In: HEILBORN, M. L. (Org.). Sexualidade: o olhar das ciências sociais. Rio de Janeiro: Zahar, 1999. p. 21-30.

ESHER, Angela; COUTINHO, Thiago. "Uso racional de medicamentos, farmaceuticalização e usos do metilfenidato”. In: Ciência e Saúde Coletiva, v. 22, n. 8, p. 2571-2580, 2017.

FARAH, Martha. "The unknowns of cognitive enhancement. Can science and policy catch up with practice?" In: Science, v. 350, n. 6259, p. 379380, 2015.

FIORE, Maurício. Uso de "drogas": controvérsias médicas e debate público. Campinas, SP: Mercado de Letras, 2006.

FLEISCHER, Soraya. "Uso e circulação de medicamentos em um bairro popular urbano na Ceilândia”, DF. In: Saúde e Sociedade, v. 21, n. 2, p. 410-423, 2012.

FOUCAULT, Michel. História da sexualidade I: a vontade de saber. Rio de Janeiro: Edições Graal, 2006 [1976].

FORLINI, Cynthia; GAUTHIER, Serge; RACINE, Eric. "Should physicians prescribe cognitive enhancers to healthy individuals?". In: Canadian Medical Association Journal, v. 185, n. 12, p. 1047-1050, 2013.

FOX, Nick; WARD, Katie. "Pharma in the bedroom and the kitchen. The pharmaceuticalisation of daily life". Sociology of Health \& Ilness, v. 30, n. 6, p. 856-868, 2008.

FREITAS, Fernando.; AMARANTE, Paulo. Medicalização em psiquiatria. Rio de Janeiro: Fiocruz, 2015.

GAUDENZI, Paula. "Mutações biopolíticas e discursos sobre o normal: atualizações foucaultianas na era biotecnológica”. In: Interface (Botucatu), v. 21, n. 61, p. 99-110, 2017.

GIURGEA, Corneliu. "The nootropic concept and its prospective implications". In: Drug Development Research, v. 2, n. 5, p. 441-446, 1982.

HARDON, Annita; SANABRIA, Emilia. "Fluid drugs: revisiting the anthropology of pharmaceuticals". In: Annu. Rev. Anthropol., v. 46, p. 117-132, 2017.

LABATE, Beatriz. et al. Drogas, políticas públicas e consumidores. Campinas, SP: Mercado de Letras, 2016.

LÉVI-STRAUSS, Claude. "A eficácia simbólica". In: Antropologia estrutural. Rio de Janeiro: Tempo Brasileiro, 1996 [1949].

LOPES, Noémia; RODRIGUES, Carla. 
"Medicamentos, consumos de performance e culturas terapêuticas em mudança”" In: Sociologia, Problemas e Práticas, Lisboa, n 78, p. 9-28, 2015.

MIGUELOTE, Vera Regina; CAMARGO JUNIOR, Kenneth Rochel. "Indústria do conhecimento: uma poderosa engrenagem". In: Revista de Saúde Pública, v. 44, n. 1, p. 190-196, 2010.

ORTEGA, Francisco. et al. "Ritalin in Brazil: production, discourse and practices". In: Interface - Comunicação, Saúde, Educação,v. 14, n. 34, p. 499-510, 2010.

PEREIRA NETO, André et al. "O paciente informado e os saberes médicos: um estudo de etnografia virtual em comunidades de doentes no Facebook". In: História, Ciências, Saúde Manguinhos, Rio de Janeiro, v. 22, supl., p. 1653 1671, 2015.

PIGNARRE, Philippe. O que é um medicamento? Um objeto estranho entre ciência, mercado e sociedade. São Paulo, Ed. 34, 1999.

ROHDEN, Fabíola. "Vida saudável versus vida aprimorada". In: Horizontes Antropológicos, v. 23, n. 47, p. 29-60, 2017.

ROSE, Nikolas. A política da própria vida: biomedicina, poder e subjetividade no século XXI. São Paulo: Paulus, 2013 [2007].

SAHAKIAN, Barbara; MOREINZAMIR, Sharon. "Pharmacological cognitive enhancement: treatment of neuropsychiatric disorders and lifestyle use by healthy people". In: Lancet Psychiatry. v. 2, n. 4, p. 357- 362, 2015.

SÁ-SILVA, Jackson Ronie et al. "Pesquisa documental: pistas teóricas e metodológicas". In: Revista Brasileira de História \& Ciências Sociais, v. 1, n. 1, jul. 2009.

TURATO, Egberto. "Métodos qualitativos e quantitativos na área da saúde: definições, diferenças e seus objetos de pesquisa”. In: Rev Saúde Pública; 39 (3):507-14, 2005.

VARGAS, Eduardo. "Fármacos e outros objetos sócio-técnicos: nota para uma genealogia das drogas". In: LABATE, B. C. et al. (Org.). Drogas e cultura: novas perspectivas. Salvador: EDUFBA, 2008.

VÍCTORA, Ceres et al. Pesquisa qualitativa em saúde: uma introdução ao tema. Porto Alegre: Tomo Editorial, 2000.

WADE, Lucie; FORLINI, Cynthia; RACINE, Eric. "Generating genius: how an Alzheimer's drug became considered a 'cognitive enhancer' for healthy individuals". In: BMC Medical Ethics, v. 15, n. $37,2014$.

WILLIAMS, Simon; MARTIN, Paul; GABE, Jonathan. "The pharmaceuticalisation of society? A framework for analysis". In: Sociology of Health \& Ilness, v. 33, n. 5, p. 1-16, 2011.

\section{Fontes consultadas}

BBC. Britânico relata 'pesadelo' em experiência com uso de 'pílula da inteligência'. G1, 16 jan. 2016. Disponível em: <http://g1.globo. com/bemestar/noticia/2016/01/britanico-relatapesadelo-em-experiencia-com-uso-de-pilulada-inteligencia.html>. Acesso em: 26 ago. 2017.

DROGAS que melhoram a inteligência ainda têm sérios efeitos colaterais. O Globo, Rio de Janeiro, 20 out. 2011. Disponível em: <https:// oglobo.globo.com/sociedade/saude/drogasque-melhoram-inteligencia-ainda-tem-seriosefeitos-colaterais-3081014>. Acesso em: 20 ago. 2017.

ESTUDO aponta que droga inteligente usada por universitários é segura. Época Negócios Online, 21 ago. 2015. Disponível em: <http://epocanegocios.globo.com/Informacao/ Resultados/noticia/2015/08/estudo-aponta-quedroga-inteligente-usada-por-universitarios-e- 
segura.html>. Acesso em: 24 ago. 2017.

FANTÁSTICO. G1, 05 jun. 2016. Disponível em: <http://g1.globo.com/fantastico/ edicoes/2016/06/05.html\#!v/5073292>. Acesso em: 28 ago. 2017.

\section{FERRARI, Márcio. Exercícios e} medicamentos deixam o cérebro mais eficiente? Época Negócios Online, 21 fev. 2016. Disponível em: <http://epocanegocios.globo.com/Vida/ noticia/2016/02/exercicios-e-medicamentosdeixam-o-cerebro-mais-eficiente.html>. Acesso em: 27 ago. 2017.

FERREIRA, Yuri. Miligramas por vaga. Uol TAB, 01 jan. 2017. Disponível em: <https://tab. uol.com.br/ritalina-vestibular\#miligramas-porvaga >. Acesso em: 27 ago. 2017.

FRANÇA, Breno; NADALETO, Otávio. Dossiê: o perigoso e tentador universo das Smart Drugs. VIP, 04 nov. 2015. Disponível em: <http:// vip.abril.com.br/saude-fitness/o-universo-dassmart-drugs/>. Acesso em: 25 ago. 2017.

GARATTONI, Bruno. Eu tomei a pílula da inteligência. Superinteressante, 23 jun. 2017. Disponível em: <https://super.abril.com. br/comportamento/eu-tomei-a-pilula-dainteligencia/>. Acesso em: 28 ago. 2017.

GNT SAIA JUSTA. Conhece a pílula da inteligência? Neurologista explica riscos. G1, 14 maio 2013. Disponível em: <http://gnt.globo. $\mathrm{com} /$ programas/saia-justa/materias/conhecea-pilula-da-inteligencia-neurologista-explicariscos.htm>. Acesso em: 28 ago. 2017.

GONZÁLEZ, Jaime. Nootrópicos, as 'drogas inteligentes' que são moda no Vale do Silício. R7 Notícias, Los Angeles, 26 jul. 2015. Disponível em: <http://noticias.r7.com/internacional/ nootropicos-as-drogas-inteligentes-que-saomoda-no-vale-do-silicio-26072015 >. Acesso em: 24 ago. 2017.
KORTE, Júlia. Viagra para o cérebro. Época, 23 set. 2014. Disponível em: <http://epoca. globo.com/vida/vida-util/saude-e-bem-estar/ noticia/2014/09/viagra-para-bo-cerebrob.html>. Acesso em: 22 ago. 2017.

MAMBRINI, Verônica. Cérebro turbinado: as técnicas e remédios para melhorar a mente. GQ Brasil, 25 jun. 2017. Disponível em: <http:// gq.globo.com/Corpo/Saude/noticia/2017/06/ cerebro-turbinado-tecnicas-e-remedios-paramelhorar-mente.html>. Acesso em: 27 ago. 2017.

MIRANDA, Giuliana. Jovens saudáveis usam remédios psiquiátricos para ir melhor em provas. Folha de São Paulo, São Paulo, 18 ago. 2015. Disponível em: <http://www1.folha.uol.com. br/equilibrioesaude/2015/08/1670037-jovenssaudaveis-usam-remedios-siquiatricos-para-irmelhor-em-provas.shtml>. Acesso em: 24 ago. 2017.

MORENO, Ana Carolina. Candidatos de concursos relatam uso de tarja preta para 'render mais. G1, São Paulo, 29 jul. 2012. Disponível em: <http://g1.globo.com/concursos-e-emprego/ noticia/2012/07/candidatos-de-concursosrelatam-uso-de-tarja-preta-para-render-mais. html>. Acesso em: 21 ago. 2017.

MUNIZ, Camilla. Governo pede atitude contra prescrição abusiva de remédios para crianças. Extra, Rio de Janeiro, 14 out. 2015. Disponível em: <https://extra.globo.com/ noticias/saude-e-ciencia/governo-pede-atitudecontra-prescricao-abusiva-de-remedios-paracriancas-17769173.html>. Acesso em: 25 ago. 2017.

NOGUEIRA Salvador; GARATTONI, Bruno. A pílula da inteligência. Superinteressante, 16 abr. 2011. Disponível em: <https://super.abril.com. $\mathrm{br} /$ ciencia/a-pilula-da-inteligencia/>. Acesso em: 28 ago. 2017.

NOGUEIRA, Viviane. A droga da produtividade. $O$ Globo, Rio de Janeiro, 14 
set. 2013. Disponível em: <https://oglobo. globo.com/sociedade/saude/a-droga-daprodutividade-9958230>. Acesso em: 22 ago. 2017.

POLATO, A.; TAVARES, I. Estudantes usam remédio para turbinar o cérebro. $R 7$ Notícias, 30 set. 2009. Disponível em: <http://noticias.r7.com/ educacao/noticias/estudantes-usam-remediopara-turbinar-cerebro-20090927.html>. Acesso em: 20 ago. 2017.

REBITE universitário, Trip, 03 jul. 2017. Disponível em: <http://revistatrip.uol.com. br/trip/estudantes-de-medicina-e-o-usoindiscriminado-de-ritalina-drogas-dainteligencia-saude-mental-suicidio-fmusp?utm source $=$ facebook\&utm_medium $=$ site-shareicon>. Acesso em: 28 ago. 2017.

SEGUNDO homem mais inteligente do mundo ingere 50 pílulas por dia para pensar melhor. Época Negócios Online, 03 dez. 2014. Disponível em: <http://epocanegocios.globo. com/Informacao/Dilemas/noticia/2014/12/ segundo-homem-mais-inteligente-do-mundoingere-50-pilulas-por-dia-para-pensar-melhor. html>. Acesso em: 23 ago. 2017.

SCHWARZ, Alan. A ascensão da pílula da boa-nota. Folha de São Paulo, São Paulo, 25 jun. 2012. Disponível em: < http://www1.folha.uol. com.br/fsp/newyorktimes/50768-a-ascensaoda-pilula-da-boa- 